\title{
Glucocorticoid-induced activation of caspase-8 protects the glucocorticoid-induced protein Gilz from proteasomal degradation and induces its binding to SUMO-1 in murine thymocytes
}

\author{
DV Delfino*, ${ }^{*}$, Spinicelli ${ }^{1}$, N Pozzesi ${ }^{1}$, S Pierangeli ${ }^{1}$, E Velardi ${ }^{1}$, S Bruscoli ${ }^{1}$, MP Martelli ${ }^{2}$, V Pettirossi ${ }^{2}$, L Falchi ${ }^{3}$, T-b Kang ${ }^{4}$ \\ and C Riccardi ${ }^{1}$
}

In this study, we evaluated the possible cross-talk between glucocorticoid (GC)-induced leucine zipper (Gilz) and caspase-8 in dexamethasone (Dex)-treated thymocytes. We determined that expression of Dex-induced Gilz protein was reduced when caspase-8 activity was inhibited, and this effect was not partially due to altered Gilz mRNA expression. Inhibition of the proteasome abrogated this reduction in Gilz expression, suggesting that Dex-induced caspase-8 activation protects Gilz from degradation. We hypothesized that the caspase-8-dependent protection of Gilz could be due to caspase-8-driven sumoylation. As a putative small ubiquitin-like modifier (SUMO)-binding site was identified in the Gilz sequence, we assessed whether SUMO-1 interacted with Gilz. We identified a 30-kDa protein that was compatible with the size of a Gilz-SUMO-1 complex and was recognized by the anti-SUMO-1 and anti-Gilz antibodies. In addition, Gilz bound to SUMO ubiquitin-conjugating (E2)-conjugating enzyme Ube21 (Ubc9), the specific SUMO-1 E2-conjugating enzyme, in vitro and coimmunoprecipitated with Ubc9 in vivo. Furthermore, Gilz coimmunoprecipitated with SUMO-1 both in vitro and in vivo, and this interaction depended on caspase-8 activation. This requirement for caspase-8 was further evaluated in caspase-8-deficient thymocytes and lymphocytes in which Gilz expression was reduced. In summary, our results suggest that caspase-8 activation protects Gilz from proteasomal degradation and induces its binding to SUMO-1 in GC-treated thymocytes.

Cell Death and Differentiation (2011) 18, 183-190; doi:10.1038/cdd.2010.86; published online 30 July 2010

The role of glucocorticoids (GCs) in regulating thymic apoptosis and development remains controversial. Apoptosis has a crucial role in the thymus as it controls the immune repertoire by selective elimination of unwanted thymocytes. ${ }^{1}$ Thymocyte apoptosis is regulated by a number of different agents, including GC and T-cell receptor (TCR)-mediated signaling. Most models postulate that the strength of TCR signaling determines thymocyte fate. In the 'mutual antagonism' model, this function is assigned to the interplay between GC- and TCR-induced apoptosis. Signaling through the TCR or GC receptor (GR) independently induces apoptosis, but when both receptors are simultaneously activated, they exhibit opposing functions. ${ }^{1-3}$

One hallmark of GC-induced thymic apoptosis is de novo protein synthesis. ${ }^{1,4,5}$ The product of the Tsc22D3 gene, GC-induced leucine zipper (Gilz), appears to be a likely candidate for GC transcriptionally induced expression that could promote thymic apoptosis. ${ }^{6}$ Tsc22D is located on chromosome $X^{7}$ The human GILZ promoter includes six GC-responsive elements (GREs). ${ }^{8-10}$ Transcriptional regulation of GILZ by GC involves the interaction of GR with GRE sequences. Three of the four sequences, which are located in the distal 5'-region of the GILZ locus, are functionally active and may require the Forkhead Box class $\mathrm{O} 3$ (FoxO3)-binding site and the 160 cofactor transcriptional intermediary factor 2 (p160 TIF2/GRIP1)-binding site. , $^{8,11-13}$

Gilz mediates several GC functions, such as modulation of T-lymphocyte activation, IL-2 production, apoptosis, and cell proliferation. ${ }^{14-16}$ Owing to the variety of protein interactions and its abundant expression in several cell types, Gilz has a crucial role in controlling protein trafficking and signaling. Recently, novel functions have been identified for Gilz, including regulation of T-helper cell differentiation ${ }^{17,18}$ and dendritic cell function, ${ }^{19,20}$ increase in renal epithelial $\mathrm{Na}^{+}$ channel (ENaC)-mediated $\mathrm{Na}^{+}$transport, ${ }^{21,22}$ and control of malignant transformation through inhibition of Ras-driven

\footnotetext{
${ }^{1}$ Section of Pharmacology Toxicology and Chemotherapy, Department of Clinical and Experimental Medicine, University of Perugia, Perugia, Italy; ${ }^{2}$ Section of Hematology, Department of Clinical and Experimental Medicine, University of Perugia, Perugia, Italy; ${ }^{3}$ Section of Onco-hematology, Department of Clinical and Experimental Medicine, University of Perugia, Perugia, Italy and ${ }^{4}$ Department of Biological Chemistry, The Weizmann Institute of Science, Rehovot, Israel ${ }^{*}$ Corresponding author: DV Delfino, Section of Pharmacology, Toxicology and Chemotherapy, Department of Clinical and Experimental Medicine, University of Perugia, Via del Giochetto, 06122 Perugia, Italy. Tel: + 39 755857467; Fax: + 39 755857405; E-mail: midelfin@ unipg.it

Keywords: GC; caspase-8; SUMO-1; Gilz; thymocyte; apoptosis

Abbreviations: GC, glucocorticoid; Gilz, glucocorticoid-induced leucine zipper; Dex, dexamethasone; TCR, T-cell receptor; GR, glucocorticoid receptor; GRE, glucocorticoid-responsive element; FoxO3, Forkhead Box class O3; p160 TIF2/GRIP1, p160 cofactor transcriptional intermediary factor 2; ENaC, epithelial Na ${ }^{+}$ channel; TAT, transactivator of transcription; E1, ubiquitin-activating enzyme; E2, ubiquitin-conjugating enzyme; E3, ubiquitin ligase; UBL, ubiquitin-like protein; SUMO, small ubiquitin-like modifier; Ubc9, SUMO E2-conjugating enzyme Ube21; Gilz-GST, Gilz bound to GST; PHA, phytohemagglutinin

Received 13.11.09; revised 04.6.10; accepted 14.6.10; Edited by JA Cidlowski; published online 30.7.10
} 
tumorigenesis. ${ }^{16}$ Strong evidence for a role of Gilz in thymic apoptosis is provided by experiments with Gilz transgenic mice, which overexpress Gilz in the T-cell lineage. Thymocytes from these mice undergo apoptosis through downregulation of $\mathrm{Bcl}-\mathrm{xL}$ and activation of caspase-8 and $-3,{ }^{15}$ suggesting that Gilz exerts effects similar to those mediated by $\mathrm{GCs}^{23}$ In addition, we have recently shown that Gilz inhibits thymocyte apoptosis induced by TCR activation, possibly by inhibiting NF-kB activity and IL-10 production. ${ }^{24}$ Thus, this response resembles the GC-like mechanism of mutual exclusion. ${ }^{23}$ In contrast, Gilz does not induce apoptosis in mature mouse T-cell lymphocytes, as a fusion protein expressing the transactivator of transcription (TAT) peptide, Gilz-TAT, triggers apoptosis in thymocytes but not in splenocytes. $^{15}$

The ubiquitin/proteasome pathway is an important system involved in the maintenance of cellular homeostasis. ${ }^{25}$ The $26 \mathrm{~S}$ proteasome is the major extralysosomal machinery used to degrade cellular proteins. It is responsible for the ATPdependent degradation of proteins tagged for destruction by ubiquitin, as well as nonubiquitinated substrates, into small peptides and amino acids. The ubiquitination pathway consists of ubiquitin-activating (E1), ubiquitin-conjugating (E2), and ubiquitin ligase (E3) enzymes. Proteasome has an important role in the regulation of multiple cellular processes, including cell-cycle progression and apoptosis. In addition to ubiquitin, there are several ubiquitin-like proteins (UBLs), such as small ubiquitin-like modifier (SUMO), which has been shown to covalently modify a large number of proteins using enzymatic machinery similar to the ubiquitination machinery. Evidence suggests that SUMO and ubiquitin may directly compete for modification of lysines in target proteins, resulting in inhibition of each other's binding to targets. ${ }^{26}$

As it has been shown that Gilz is involved in GC-induced thymocyte apoptosis and this is known to be a caspasedependent process, the goal of this study was to further evaluate the relationship between Gilz and the caspase cascade. We determined that proteasomal degradation of Gilz increases if caspase-8 activation is inhibited during GC-induced thymocyte apoptosis. In contrast, when the apoptotic machinery is active, Gilz protein is expressed and covalently binds to SUMO-1.

\section{Results}

Effect of caspase-8 inhibition on Dex-induced Gilz expression in thymocytes. We previously determined that Gilz promotes thymocyte apoptosis through the downregulation of $\mathrm{Bcl}-\mathrm{xL}$, and this is associated with activation of caspases- 8 and -3 , but not caspase- 9 activation. To further examine the relationship between Gilz and caspase8, we analyzed Gilz expression when the activation of either caspase- 8 or -9 was inhibited. To do this, thymocytes from normal mice were stimulated with dexamethasone (Dex), a synthetic GC, and either caspase-8 or -9 activation was inhibited using the Z-IETD and Z-LEHD chemical inhibitors, respectively. After $6 \mathrm{~h}$ of culture, the total proteins were extracted and Gilz expression was analyzed by western blotting. As shown in Figure 1a, inhibition of caspase-8, but

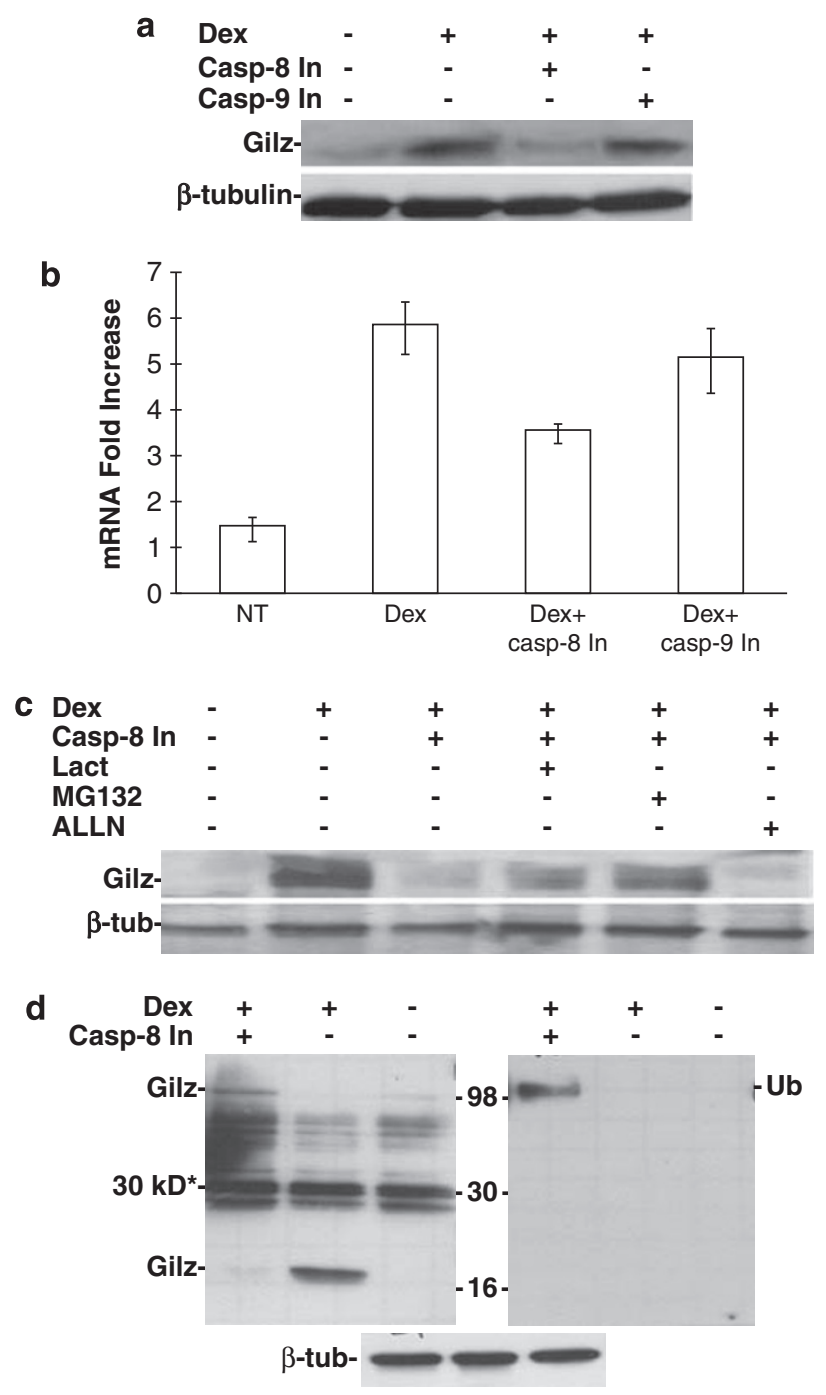

Figure 1 Effect of caspase-8 inhibition on Gilz protein expression. (a) Western blotting analysis of the total protein extracted from thymocytes cultured for $6 \mathrm{~h}$ : untreated (lane 1); treated with $10^{-7} \mathrm{M}$ dexamethasone (Dex, lane 2); Dex plus Z-IETD-FMK (75 $\mu \mathrm{M}$ ), a caspase-8 inhibitor (Casp-8 In, lane 3); and Z-LEHD-FMK $(75 \mu \mathrm{M})$, a caspase-9 inhibitor (Casp-9 In, lane 4). Bands indicate Gilz (upper panel) or $\beta$-tubulin (lower panel) expression. (b) Real-time PCR of thymocytes treated for $3 \mathrm{~h}$ as described in panel a. Bars indicate the fold increase in Gilz mRNA \pm 1 S.D. (c) Western blotting analysis of proteins isolated from thymocytes: untreated (lane 1), treated with Dex alone (lane 2) or Dex plus Z-IETD-FMK (Casp-8 In, lane 3), plus Z-IETD-FMK and lactacystin (Lact, lane 4, $10 \mu \mathrm{M}$ ), MG132 (lane 5, $10 \mu \mathrm{M}$ ), or ALLN (lane 6, $10 \mu \mathrm{M}$ ). (d) Western blotting analysis of proteins isolated from thymocytes: treated with Dex and Z-IETD-FMK (Casp-8 In, lane 1), treated with Dex alone (lane 2) or untreated (lane 3). The filter was probed for Gilz (upper left), ubiquitin ( $\mathrm{Ub}$, upper right) or $\beta$-tubulin ( $\beta$-tub, lower). Numbers indicate the protein standard $(\mathrm{kDa})$, and the asterisk indicates the nonspecific $30-\mathrm{kDa}$ band. All data are from three independent experiments, except $\mathbf{d}$, which was performed once

not caspase-9, reduced the Dex-mediated induction of Gilz protein expression. In agreement with previously reported data from our group, ${ }^{4}$ Dex treatment induced a high percentage of thymocyte apoptosis (about $80 \%$ ), which was almost completely inhibited (to about 12\%) along with the downregulation of Gilz by Z-IETD, but only slightly (to about $51 \%$ ) by Z-LEHD (Supplementary Figure 1). 
To determine whether this reduction in expression was due to inhibition of Gilz mRNA transcription, real-time PCR was performed in thymocytes under the same treatment conditions as in Figure 1a. As shown in Figure 1b, inhibition of caspase- 8 activity decreased only by $\sim 50 \%$. The Gilz mRNA expression was much higher than the untreated control group, which also exhibited a similar lack of Gilz protein expression. These results suggest that the abrogation of Gilz protein expression by inhibition of caspase- 8 activity is partially due to either abrogation of mRNA translation or increased protein degradation.

The role of protein degradation was assessed by inhibiting the two branches of cell proteolysis: (1) the proteasome and (2) the lysosomes. Thymocytes stimulated with Dex and treated with the caspase-8 inhibitor were concomitantly treated with lactacystin or MG-132, which are irreversible and reversible proteasomal inhibitors, respectively, or ALLN, a lysosomal inhibitor. The reduction in Dex-induced Gilz protein expression in the presence of the caspase- 8 inhibitor was partially reversed by treatment with lactacystin and MG-132 but not with ALLN (Figure 1c). These results suggest that the reduction in Dex-induced Gilz protein expression by inhibition of caspase- 8 is partly due to increased proteasomalmediated degradation, and the partial restoration of Gilz protein by MG132 parallels the partial reduction in mRNA expression induced by Z-IETD treatment.

As proteins are targeted for proteasomal degradation by the binding of polyubiquitin, we performed western blotting to visualize whether binding of polyubiquitin when caspase-8 activation was inhibited in Dex-treated thymocytes. As shown in Figure 1d, expression of polyubiquinated proteins increased in cells in which caspase- 8 activation was inhibited, as compared with untreated or Dex-treated thymocytes. The same filter was probed for Gilz expression (Figure 1d, left panel). Gilz was expressed in the Dex-treated thymocytes in its native form of $17 \mathrm{kDa}$, whereas in the cells treated with the caspase-8 inhibitor, there was no expression of the $17-\mathrm{kDa}$ form. In these treated cells, a heavy molecular weight form of Gilz migrated at the same size as the polyubiquitinated proteins. These results indicate that inhibition of caspase-8 activation is associated with the polyubiquitination of Gilz, which results in increased activity of the proteasomal degradation pathway.

Evaluation of whether Gilz can interact with SUMO-1. As inhibition of Dex-induced caspase-8 cleavage activated the ubiquitin-proteasomal pathway, it is reasonable to propose that Dex-induced activation of caspase-8 protects Gilz from polyubiquitination and proteasomal degradation. It has been extensively reported in the literature that the binding of certain target proteins, such as NF- $k B$, to ubiquitin is inhibited by the binding of SUMO-1 to the same target lysine. ${ }^{26,27}$ A potential high-scoring sumoylation-binding site was identified in the Gilz protein sequence (Figure 2a), and so it was reasonable that the binding of either SUMO-1 or ubiquitin to Gilz could ultimately determine whether Gilz was expressed or degraded, respectively.

If SUMO-1 covalently binds to Gilz, this would result in a protein complex of $\sim 30 \mathrm{kDa}$ as Gilz is $17 \mathrm{kDa}$ and SUMO-1 is $11 \mathrm{kDa}$. To assess this possibility, western blotting was performed on total protein extracts from untreated or Dex-treated thymocytes. As Figure $2 b$ shows, the Gilz antibody (Ab) recognized both a $17-\mathrm{kDa}$ and a $30-\mathrm{kDa}$ protein, and both these bands were upregulated in the Dex-treated thymocytes, as compared with untreated thymocytes. In the same samples, the anti-SUMO-1 Ab recognized a band of $\sim 30 \mathrm{kDa}$, and expression of this protein was greater in Dex-treated thymocytes than in untreated cells. These results suggest that covalent binding of SUMO-1 to Gilz in Dex-treated thymocytes is a plausible hypothesis and should be further evaluated.
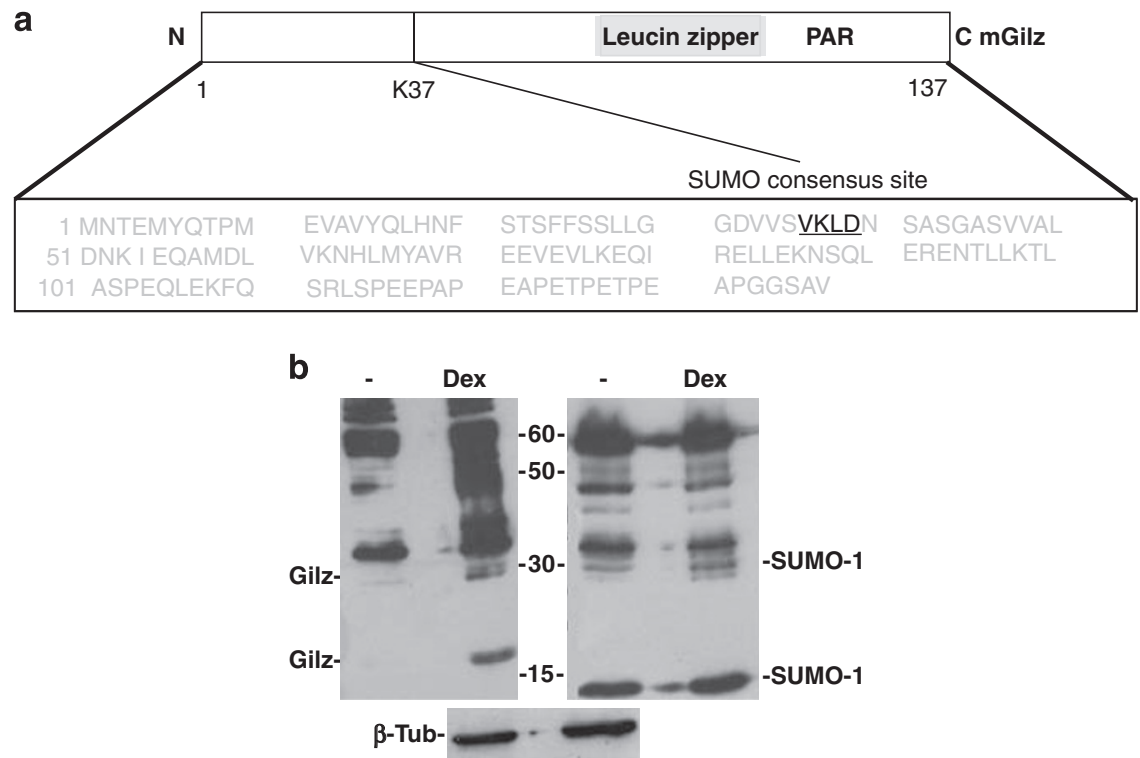

Figure 2 Expression of SUMO-1 in Dex-treated thymocytes. (a) Schematics of protein (upper) and amino acid (lower) sequences for murine Gilz (mGilz). The SUMO consensus site is indicated in black. (b) Western blotting analysis of total protein extracted from thymocytes cultured for $6 \mathrm{~h}$ : untreated (lane 1) or treated with $10^{-7}$ dexamethasone (Dex, lane 2) and probed for Gilz (left) or SUMO-1 (right). Data are from three independent experiments 
Gilz binds to Ubc9. The covalent binding of SUMO-1 to target proteins is a well-established process known to function similarly to ubiquitination. It involves three key enzymes (E1, E2, and E3). SUMO E2-conjugating enzyme Ube21 (Ubc9) is believed to be the only SUMO-1-conjugating enzyme, ${ }^{26,27}$ and so it is useful to assess whether a target protein is sumoylated by examining whether it is bound to Ubc9. We used two strategies to assess the binding of Gilz to Ubc9: an in vitro pull-down binding assay and Ubc9 immunoprecipitation. Ubc9 was expressed in thymocytes irrespective of any treatment with Dex or the caspase-8 inhibitor (Figure 3a, right panel). Gilz bound to GST (GilzGST) only pulled down Ubc9 in thymocytes that were treated with Dex but not in untreated or Dex-treated thymocytes that were concomitantly treated with the caspase-8 inhibitor (Figure 3a, left panel). As shown in Figure 3b, Ubc9 was expressed in both the untreated and Dex-treated thymocytes (upper panel, left bands), and the anti-Ubc9 Ab immunoprecipitated Ubc9 from both these cells (upper panel,

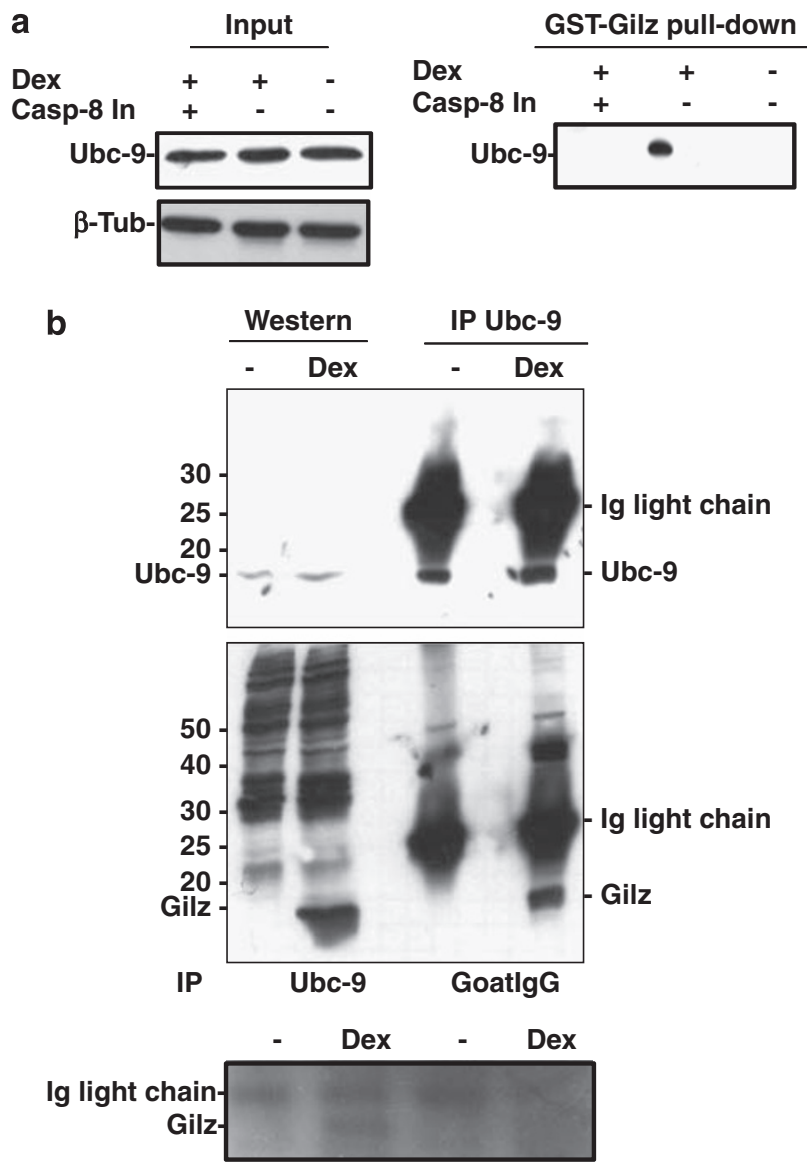

Figure 3 Binding of Gilz to Ubc9. (a) In vitro pull-down experiments. On the left, proteins isolated from thymocytes treated for $6 \mathrm{~h}$ with $10^{-7} \mathrm{M}$ Dex plus $75 \mu \mathrm{M}$ Z-IETD-FMK (Casp-8 In, lane 1), Dex alone (lane 2) or untreated (lane 3), exposed to GST-Gilz fusion protein and probed for Ubc9 expression. On the right, western blotting of the same samples probed for Ubc9. (b) Upper panel: on the left, western blotting of proteins isolated from untreated or Dex-treated thymocytes probed for Ubc9; and on the right, the same protein samples subjected to immunoprecipitation with anti-Ubc9 Ab. The lower panel is the same filter probed for Gilz. Experiments were performed three times and representative results are provided right bands). In the lower panel (left bands), western blotting confirms that Gilz was only expressed in the thymocytes treated with Dex, and immunoprecipitation of lysates with the Ubc9 Ab (right bands) coimmunoprecipitated Gilz in Dextreated thymocytes. This coimmunoprecipitation is specific as there was no binding of Gilz observed when the IgG isotype control was used. These results suggest that Ubc9 binds to Gilz in vitro and in vivo, and as Ubc9 is the specific E2 that mediates the binding of SUMO-1 to target proteins, this suggests that Gilz is sumoylated in thymocytes treated with Dex that have activated caspase-8.

Gilz binds to SUMO-1. To directly confirm that the $30-k D a$ band represented Gilz bound to SUMO-1, we used two strategies. First, in vitro pull-down experiments with Gilz-GST were performed to determine whether Gilz could bind to SUMO-1 in vitro. As shown in Figure 4a (left panel), SUMO-1 was expressed in untreated thymocytes, thymocytes treated with Dex alone, and thymocytes concomitantly treated with Dex plus either the caspase-8 or -3 inhibitor. We used the caspase- 3 inhibitor as a control because caspase- 3 is activated downstream of caspase- $8 ;{ }^{4}$ therefore, in cells in which caspase- 3 is inhibited, caspase- 8 is activated. When the protein lysates from these samples were subjected to pull down with GST-Gilz, SUMO-1 bound to Gilz in the cells with activated caspase-8 (Dex alone and Dex plus caspase-3 inhibitor), but not in the cells in which caspase-8 was inactive (Dex plus caspase-8 inhibitor). SUMO-1 bound to GST-Gilz

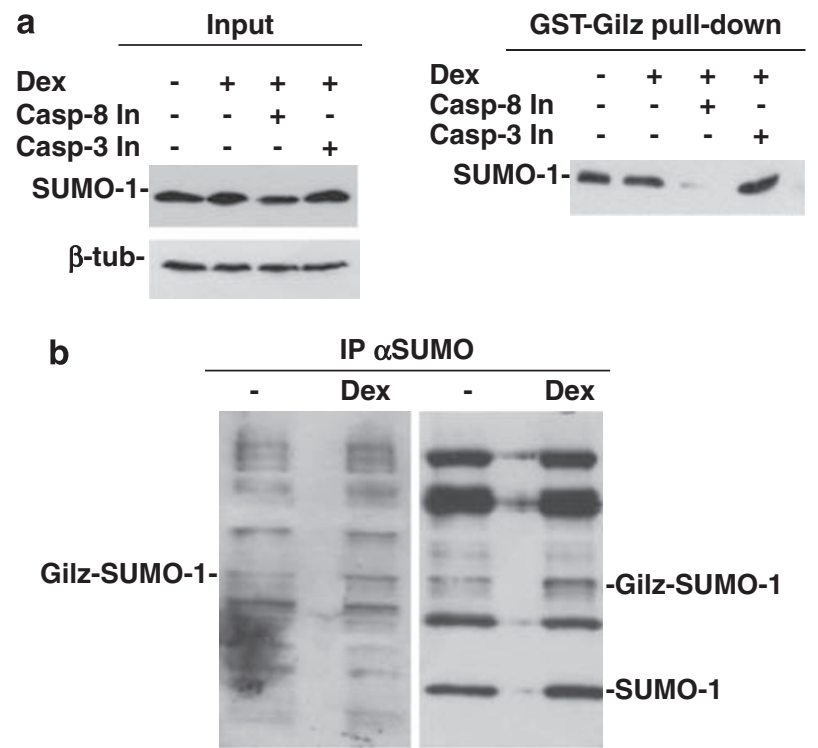

Figure 4 Binding of Gilz to SUMO-1. (a) Left: western blotting of proteins from thymocytes cultured for $6 \mathrm{~h}$ : untreated (lane 1); treated with $10^{-7} \mathrm{M}$ Dex alone (lane 2); or Dex plus $75 \mu \mathrm{M}$ Z-DEVD-FMK (caspase-3 inhibitor, Casp-3 In, lane 3); or $75 \mu \mathrm{M}$ Z-IETD-FMK (Casp-8 In, lane 4) and probed for SUMO-1 expression. Right: same samples subjected to pull-down with a GST-Gilz fusion protein and probed for SUMO-1. (b) Immunoprecipitation with anti-SUMO-1 Ab of protein lysates from untreated (lane 1) or Dex-treated (lane 2) thymocytes. The same filter was probed for SUMO-1 (right panel) or for Gilz (left panel). Experiments were performed three times and representative results are provided 
in untreated thymocytes possibly as a consequence of basal caspase-8 activation; however, SUMO-1 binding to Gilz in the absence of Dex is not a consistent finding (Figure $4 a$, right panel).

Second, coimmunoprecipitation studies were performed with the untreated and Dex-treated thymocyte lysates. The samples were subjected to immunoprecipitation with an antiSUMO-1 Ab, followed by western blotting with an anti-Gilz Ab. As shown in Figure 4b, SUMO-1 covalently bound to Gilz in the untreated cells and with increased affinity in Dex-treated thymocytes.

Role of caspase-8. These results suggested that Dexinduced activation of caspase- 8 has a role in the sumoylation of Gilz either directly or indirectly. To further investigate this, we assessed Gilz expression in thymocytes isolated from caspase-8-floxed mice either untreated or treated with Dex and cultured for $6 \mathrm{~h}$. These mice, developed in the laboratory of Dr Wallach, ${ }^{28,29}$ exhibit a strong downregulation of caspase- 8 in their thymocytes (Figure $5 \mathrm{a}$, left panel). This reduction parallels the decrease (about 50\%) in Gilz expression observed in both untreated and Dex-treated thymocytes (Figure 5a, middle panel, quantization on the right). The role of caspase- 8 was also analyzed in the caspase-8-defective I 9.2 human cell line. This is a mutant cell line derived from the A3 cell line, a clone of the Jurkat cell line that is hyperresponsive to Fas-mediated apoptosis. The A3 cell line is unresponsive to $\mathrm{GC}$, but activates caspase-8 upon phytohemagglutinin (PHA) treatment. ${ }^{30}$ Thus, A3 and I 9.2 cells were treated with PHA $(10 \mu \mathrm{g} / \mathrm{ml})$ for $16 \mathrm{~h}$, and caspase- 8 activation and Gilz expression were evaluated by western blotting. As shown in Figure $5 a$ (lower panel), caspase- 8 was expressed and activated after PHA treatment in A3 cells, whereas, as expected, it was not expressed in I 9.2 cells. PHA treatment induced Gilz expression in A3 cells but its expression in I 9.2 cells was decreased to $\sim 50 \%$ as shown by quantification (right panel). The remaining expression of Gilz, which was expressed despite the absence of caspase-8, could have been due to caspase-10 expression. This caspase is very similar, both structurally and functionally, to caspase-8 and is only expressed in human cells and not in murine cells. To provide insight into the possible mechanism for this effect, we tested whether Gilz could directly bind to caspase-8 using immunoprecipitation. We transfected COS-7 cells with myc-tagged Gilz and subjected the cell lysates to caspase- 8 immunoprecipitation.

a
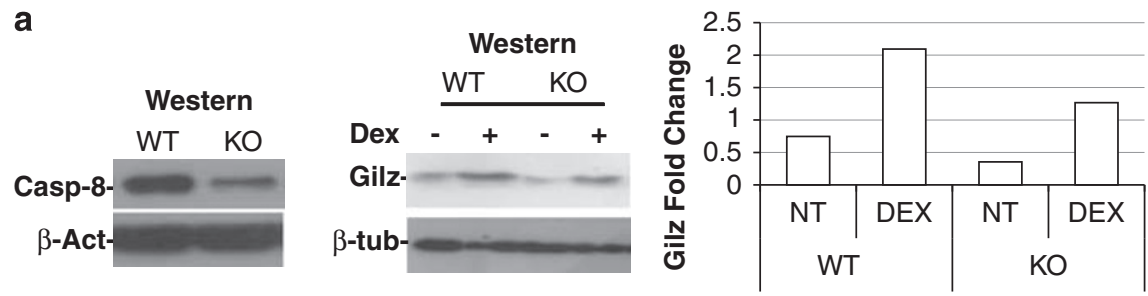

b
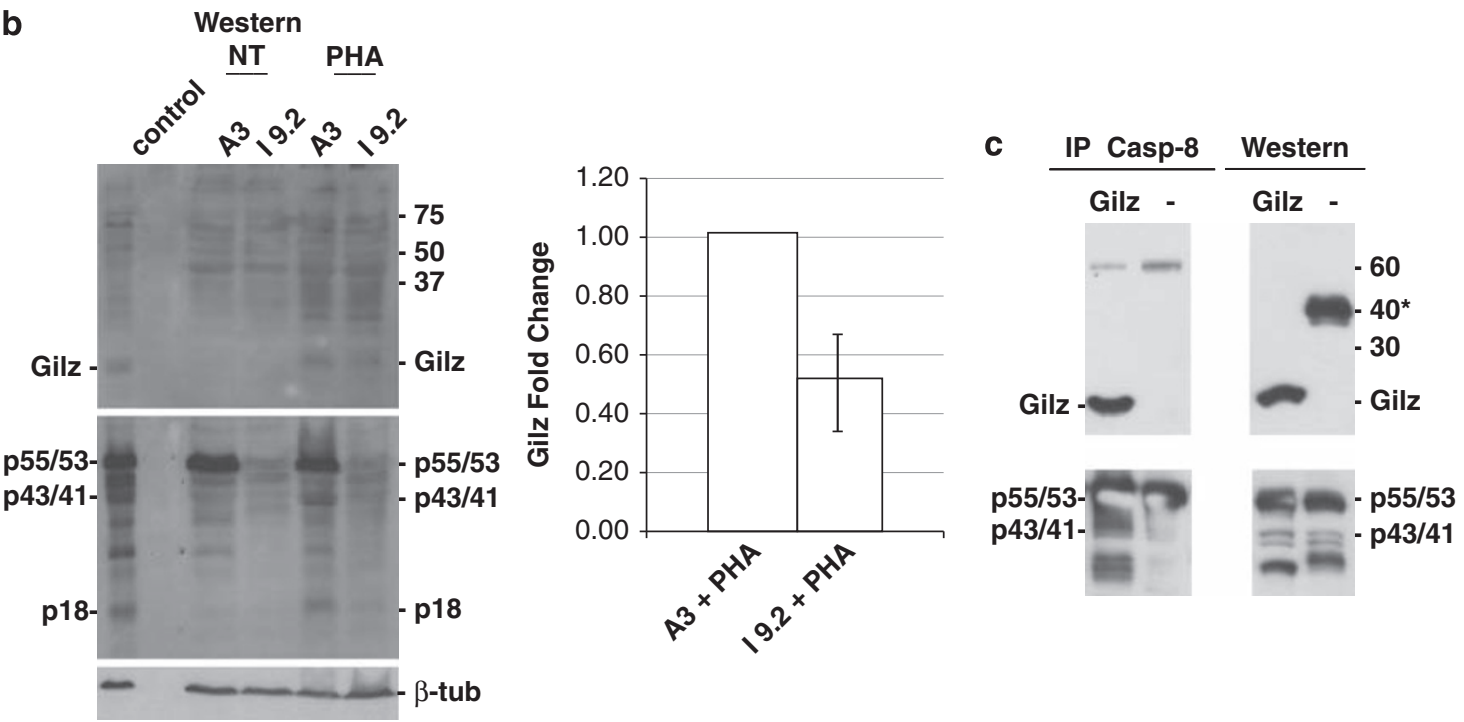

Figure 5 Expression of Gilz in caspase-8-deficient cells. (a) Western blot of thymocyte proteins extracted from WT and caspase-8-floxed mice. The left panel shows the expression of caspase-8; the middle panel the expression of Gilz in untreated and Dex-treated thymocytes (6 h culture); and on the right its quantization. (b) Western blotting analysis of proteins from A3 (caspase-8 functional) or I 9.2 (caspase-8 deficient) cells untreated or treated for $16 \mathrm{~h}$ with PHA (10 $\mu \mathrm{g} / \mathrm{ml}$ ) and probed for Gilz expression (upper panel), caspase-8 (p55/53, uncleaved; $\mathrm{p} 43 / 41$, partially cleaved; or p18, cleaved (middle panel) or $\beta$-tubulin ( $\beta$-tub, lower panel). The right panel is the quantification from two independent experiments. (c) Immunoprecipitation with anti-caspase-8 Ab of protein lysates from COS-7 cells transfected with myc-tagged Gilz. Upper left: western blot with anti-myc Ab. Upper right: western blotting on the same samples probed with Gilz (asterisk indicates the presence of an unknown band). Lower left: same filter as in the upper left panel probed with anti-caspase-8 Ab (p55/53, uncleaved; $\mathrm{p} 43 / 41$, partially cleaved). Lower right: same filter as in upper right panel reprobed for caspase-8. Experiments were performed three times and representative results are provided 
The immunoprecipitation was successful (Figure 5c); caspase- 8 was found to be bound to myc-tagged Gilz (Figure 5, upper panel). These results suggest that Gilz expression depends on the expression and activation of caspase-8, which confirms our previous conclusions. This latter result suggests that the effect of caspase-8 activation on Gilz protein expression may be direct. The conclusion that caspase-8 (or other requirements) is critical for sumoylation of Gilz may be indirectly inferred by the results of an in vitro sumoylation assay. As shown in Supplementary Figure 2, the sumoylation of the fusion protein GST-Gilz did not occur, suggesting that additional components (for example, a novel $E_{3}$-ligating enzyme) are required for this reaction to proceed. We hypothesize that these additional requirements are dependent on caspase-8 activation, but this hypothesis requires further experimentation to be confirmed.

\section{Discussion}

GCs induce thymocyte apoptosis. ${ }^{31}$ Although GR-mediated gene activation is clearly an essential component of the apoptotic pathway, ${ }^{32}$ the downstream events are controversial. Although GC-induced cell death does not directly proceed through one of the two classical apoptotic pathways, $\mathrm{Bcl}-2$ proteins and caspases do appear to be involved in this process. ${ }^{33-35}$ The involvement of caspases in GC-induced apoptosis is continually debated. By using pharmacological inhibitors, caspase- 3 and -8 were proposed to mediate GC-induced apoptosis, although their roles seem to be cell type specific. ${ }^{4,31}$ In contrast, thymocytes derived from a $T$ lymphocyte with specific deletion of caspase-8 show no differences from wild-type thymocytes in terms of responsiveness to $\mathrm{GC}$ treatment. ${ }^{36} \mathrm{GC}$ also induces the expression of Gilz in thymocytes, ${ }^{6}$ and Gilz itself promotes thymocyte apoptosis, downregulation of $\mathrm{Bcl}-\mathrm{xL}$, and activation of caspase-8. ${ }^{15}$ This suggests that Gilz may be involved in GC-mediated thymocyte apoptosis, although there are no conclusive reports on this functional relationship. The relationship between Gilz and caspase-8 evokes several questions. Are these biochemical events consequentially triggered or are they independent events? Are there any additional connections between Gilz and caspase-8? To answer these questions, we inhibited GC-induced activation of caspase-8 in thymocytes and evaluated the expression of Gilz protein. Caspase- 8 and -9 were inhibited using the chemical inhibitors Z-IETD and Z-LEHD, respectively. Although these caspase inhibitors lack specificity at high concentrations, ${ }^{37}$ our group has previously demonstrated that they completely inhibit the caspases and seem to be specific at the concentrations used in terms of GC-induced apoptosis. ${ }^{4}$ The specificity of caspase inhibition is a difficult issue as caspase- $8,-9$, and -3 belong to the same pathways. In GC-induced thymocyte apoptosis, activation of caspase- 8 triggers two different pathways: (1) the 'extrinsic' pathway, which directly activates caspase-3, and (2) the 'intrinsic' pathway, which releases cytochrome $c$ from the mitochondria and activation of caspase- 9 that subsequently activates caspase- 3 . This means that any specific inhibitor of caspase-8 activation also inhibits the activation of caspase- 9 and -3 . The inhibition of caspase- 9 is indirect because Z-IETD inhibits cytochrome $c$ release, which is an upstream event in caspase- 9 activation. The specific inhibition of caspase- 9 by Z-LEHD also inhibits caspase-3, but not caspase-8, whereas inhibition of caspase-3 (a terminal caspase) has no effect on activation of the two other caspases. ${ }^{4}$ Our results indicate that inhibition of caspase-8, but not caspase- 9 , induces an almost complete reduction in Gilz protein but only a partial reduction in Gilz mRNA expression. This reduction in expression was partially due to increased proteasomal degradation of the protein, as confirmed by proteasomal inhibitor experiments and the presence of a slowly migrating form of Gilz (most likely a polyubiquitinated form). The first set of experiments suggests two conclusions: (1) GCs must stimulate transcription of the target gene, in addition to protecting it from protein degradation to achieve stable protein expression; and (2) Gilz induces caspase-8 activation, which promotes the expression of Gilz, and so we can propose that a feedback loop exists between Gilz expression and caspase-8 activation.

How does caspase-8 activation protect Gilz from proteasomal degradation? One possibility suggested by numerous studies is that ubiquitin binding to the target protein is inhibited by the binding of SUMO-1 to the same target lysine. As computational analysis of the Gilz protein sequence indicated the presence of a putative SUMO-binding site at lys 37 of the Gilz protein, we assessed whether activation of caspase-8 could determine the sumoylation of Gilz. This was confirmed by three sets of data: (1) the presence of a protein band that migrated at $30 \mathrm{kDa}$, which was compatible with covalent binding of SUMO-1 (11 kDa) to Gilz $(17 \mathrm{kDa})$; (2) the involvement of the SUMO-specific E2-conjugating enzyme Ubc9, which was found to bind to Gilz both in vitro and in vivo in a caspase-8 activation-dependent manner; and (3) the binding of SUMO-1 to Gilz both in vitro and in vivo.

The presence of a 30-kDa band suggests potential covalent SUMO-1-Gilz binding but further experiments are required to confirm this interaction. In the experiment in Figure 1d, it was difficult to discriminate between proteins in the middle of the gel; thus, a 30-kDa nonspecific band may have hidden the 30$\mathrm{kDa}$ band in the Dex-treated thymocyte samples.

Sumoylation is a multistep process and requires several enzymes. Among them is the E2-conjugating enzyme Ubc9, which transfers the activated SUMO-1 to protein substrates. Although Ubc9 is ubiquitously expressed in all tissues, it transfers SUMO-1 to protein substrates only when there is previous activation of SUMO-1. ${ }^{38}$ This seemed to occur in our system: Ubc9 is constitutively expressed in thymocytes independently of their functional state but it binds to Gilz only in the case of Dex-dependent caspase-8 activation, which either directly or indirectly triggers the sumoylation pathway.

What is the relationship between Gilz proteasomal degradation/sumoylation and caspase- 8 activation? To provide insight into this question, we used a caspase-8-deficient T lymphoma cell line, I 9.2, which is a mutated clone of the A3 cell line, a clone of the Jurkat cell line. We stimulated the activation of caspase- 8 by treating cells with PHA for $16 \mathrm{~h}$ and demonstrated that PHA treatment induced activation of caspase- 8 and Gilz expression in A3 cells, whereas PHA treatment of I 9.2 cells (caspase- 8 deficient) did not induce Gilz protein expression. Thus, these results confirm the previous finding that activation of caspase- 8 affects Gilz 
expression, and following transfection of COS-7 cells with Gilz, we demonstrated that Gilz binds to caspase-8 using immunoprecipitation.

It is possible that caspase activation inhibits proteasome function, as previously reported; ${ }^{39}$ therefore, this and the results from our study evoke further questions that warrant investigation. Is the direct binding of Gilz to caspase-8 responsible for its sumoylation, or is this due to an indirect effect? Further experiments are required to dissect this issue. However, we can conclude that caspase-8 affects the sumoylation of proteins in addition to its apoptotic functions. ${ }^{40,41}$

Our new results on Gilz expression and its relationship with caspase-8 raise a number of new questions about their impact on human diseases. Specifically, the effect of Gilz on proliferation/apoptosis, and its binding to Ras makes it a likely contributor to human cancer. $^{16}$ In addition, congenital absence of caspase-8 contributes to autoimmune lymphoproliferative syndrome in which the development of autoimmune diseases (because of lack of apoptosis negative selection in the thymus) is linked to a lymphoproliferative disease that causes death in young patients. ${ }^{42}$ Is there any role for Gilz in this aberrant condition? Is it possible to treat this disease using soluble Gilz that can overcome the caspase-8 inhibition? Additional experimentation will allow clarification of the importance of Gilz in this congenital disorder and also provide insight on the relationship between autoimmune diseases and lymphoproliferative disorders.

\section{Materials and Methods}

Animals. C57BL/6 mice were purchased from Charles River (Calco, Lecco, Italy), and 3-week-old mice were used for all experiments. They were housed in an isolated colony and provided with laboratory chow and acidified (pH 2.4) water ad libitum. Caspase-8-floxed mice were generated as previously described. ${ }^{28}$ To generate $T$ cell-specific caspase-8-deficient mice, caspase-8-floxed mice were crossed with Lck-Cre transgenic mice ${ }^{29}$ (The Jackson Laboratory, Bar Harbor, MA, USA).

Cell harvesting and cell culture. Thymocytes were counted using a hemocytometer. Single thymocyte suspensions were cultured in RPMI 1640 medium containing $5 \%$ fetal calf serum, $100 \mathrm{U} / \mathrm{ml}$ penicillin/streptomycin, $0.1 \mathrm{mM}$ nonessential amino acids, and $5.5 \times 10^{2} \mu \mathrm{M} \quad \beta$-mercaptoethanol (GIBCO Invitrogen, San Giuliano Milanese, Italy). Cells were maintained in flat-bottomed, 96-well plates (Costar, Cambridge, MA, USA). The A3 and I 9.2 cell lines were obtained from American Type Tissue Culture Collection (ATCC, Manassas, VA, USA).

Reagents. The caspase inhibitors were Z-DEVD-FMK, a caspase- 3 inhibitor; Z-IETD-FMK, a caspase-8 inhibitor; and Z-LEHD-FMK, a caspase-9 inhibitor. All were purchased from Calbiochem (San Diego, CA, USA) and used at $75 \mu \mathrm{M}$. In general, cells were preincubated with the inhibitors for 30 min before Dex treatment $\left(10^{-7} \mathrm{M}\right)$. MG-132, lactacystin, and ALLN were also purchased from Calbiochem and used at $10 \mu \mathrm{M}$.

Real-time PCR analysis. Total RNA was extracted using Trizol (Invitrogen). RT-PCR was performed using QuantiTect Reverse Trascription (Qiagen, Milan, Italy). For real-time PCR, the sense primer for GILZ was $5^{\prime}$-GGTGGCCCTAGACAA CAAGA-3' ${ }^{\prime}$, and the antisense primer was $5^{\prime}$-TCTTCTCAAGCAGCTCACGA-3'. For HPRT, the sense primer was $5^{\prime}$-CGTCGTGATTAGCGATGATG- $3^{\prime}$, and the antisense primer was $5^{\prime}$-ACAGAGGGCCACAATGTGAT- ${ }^{\prime}$. PCR was performed on the Abi 7300 (Applied Biosystems, Carlsbad, CA, USA) using Power SYBR GREEN (Applied Biosystems). Relative amounts of GILZ mRNA were calculated using the comparative $\Delta \Delta C(\mathrm{t})$ method. $C(\mathrm{t})$ values were determined using the 7300 System Software (Applied Biosystems)
Western blot analysis. Cells were lysed in radio-immunoprecipitation assay buffer ( $50 \mathrm{mM}$ Tris- $\mathrm{HCl}$ pH $8,150 \mathrm{mM} \mathrm{NaCl}, 0.5 \%$ sodium deoxycholate, $0.1 \%$ SDS, $1 \% \mathrm{NP} 40$, protease inhibitor cocktail, and $20 \mathrm{mM} \mathrm{N}$-ethyl maleimide). Total proteins were separated using 12 or $15 \%$ SDS polyacrylamide gel electrophoresis (SDS-PAGE). The primary Abs were rabbit polyclonal antiserum anti-Gilz produced by our laboratory; rabbit polyclonal anti-GILZ (FL-134) (sc-33780, Santa Cruz Biotechnology, Santa Cruz, CA, USA); anti-caspase-8 (Santa Cruz Biotechnology); anti-caspase-3; anti-caspase-9 (Cell Signaling Technology, Beverly, MA, USA); goat anti-Ubc9 (N-15) (sc-5231, Santa Cruz Biotechnology); mouse monoclonal anti-SUMO-1 (mouse anti-GMP-1, clone 21C7, isotype $\operatorname{lgG}_{1}-\kappa$, Invitrogen); rabbit polyclonal anti-SUMO-1 (FL-101) (sc-9060, Santa Cruz Biotechnology); and antitubulin (Sigma, St Louis, MO, USA). The secondary Abs were horseradish peroxidase-conjugated donkey anti-goat IgG (sc-2033, Santa Cruz Biotechnology), goat anti-rabbit (Thermo Scientific, Línea Pierce, cat. 31460, Rockford, IL, USA) and the isotype control was normal goat IgG (sc-2028) (Santa Cruz Biotechnology).

In vitro protein-binding assay. Extracts were generated from untreated and Dex-treated $(100 \mathrm{nM})$ thymocytes. GST-Gilz fusion proteins were prepared, as previously described. ${ }^{15}$ Pull-down assays were performed by incubating the fusion protein, loaded onto glutathione-sepharose beads, with cellular lysates in binding buffer $(250 \mathrm{mM} \mathrm{NaCl}, 50 \mathrm{mM}$ HEPES, pH 7.5, $0.5 \mathrm{mM}$ EDTA, 0.1\% (v/v) NP-40, $0.2 \mathrm{mM}$ PMSF, $1 \mathrm{mM} \mathrm{DTT}$, and $100 \mu \mathrm{g} / \mathrm{ml} \mathrm{BSA}$ ) for $18 \mathrm{~h}$ at $4^{\circ} \mathrm{C}$. The beads were extensively washed, resuspended in sample buffer, and analyzed using SDS-PAGE and western blotting with the indicated Abs.

GST or GST-Gilz, bound to glutathione-sepharose beads, were incubated with in vitro translated proteins for $18 \mathrm{~h}$ at $4^{\circ} \mathrm{C}$. The beads were subsequently washed five times with $0.5 \mathrm{ml}$ of binding buffer. The bound proteins were analyzed using SDS-PAGE and autoradiography.

Statistical analysis. Data represent the mean \pm 1 S.E.M.

\section{Conflict of interest}

The authors declare no conflict of interest.

Acknowledgements. This work was supported in part by Associazione Italiana Ricerca Cancro (AIRC) and by the Italian Ministry of University (PRIN 2008RFPZN7_002).

1. Ashwell JD, Lu FWM, Vacchio MS. Glucocorticoids in T cell development and function. Annu Rev Immunol 2000; 18: 309-345.

2. King LB, Vacchio MS, Ashwell JD. To be or not to be: mutually antagonistic death signals regulate thymocyte apoptosis. Int Arch Allergy Immunol 1994; 105: 355-358.

3. Erlacher M, Knoflach M, Stec IE, Bock G, Wick G, Wiegers GJ. TCR signaling inhibits glucocorticoid-induced apoptosis in murine thymocytes depending on the stage of development. Eur J Immunol 2005; 35: 3287-3296.

4. Marchetti MC, Di Marco B, Cifone G, Migliorati G, Riccardi C. Dexamethasone-induced apoptosis of thymocytes: role of glucocorticoid receptor-associated Src kinase and caspase-8 activation. Blood 2003; 101: 585-593

5. Cifone MG, Migliorati G, Parroni R, Marchetti C, Millimaggi D, Santoni A et al. Dexamethasone-induced thymocyte apoptosis: apoptotic signal involves the sequential activation of phosphoinositide-specific phospholipase C, acidic sphingomyelinase, and caspases. Blood 1999; 93: 2282-2296.

6. D'Adamio F, Zollo O, Moraca R, Ayroldi E, Bruscoli S, Bartoli A et al. A new dexamethasone-induced gene of the leucine zipper family protects $T$ lymphocytes from TCR/CD3-activated cell death. Immunity 1997; 7: 803-812.

7. Cannarile L, Zollo O, D'Adamio F, Ayroldi E, Marchetti C, Tabilio A et al. Cloning, chromosomal assignment and tissue distribution of human GILZ, a glucocorticoid-induced gene. Cell Death Differ 2001; 8: 201-203.

8. Asselin-Labat ML, David M, Biola-Vidamment A, Lecoeuche D, Zennaro MC, Bertoglio J et al. GILZ, a new target for the transcription factor $\mathrm{FoxO}$, protects $\mathrm{T}$ lymphocytes from interleukin-2 withdrawal-induced apoptosis. Blood 2004; 104: 215-223.

9. Wang JC, Derynck NK, Nonaka DF, Khodabackhsh DB, Happ C, Yamamoto KR. Chromatin immunoprecipitation (ChIP) scanning identifies primary glucocorticoid receptor target genes. Proc Natl Acad Sci USA 2004; 101: 15603-15608.

10. Tynan SH, Lundeen SG, Allan GF. Celltype-specific bidirectional regulation of the glucocorticoid-induced leucine zipper (GILZ) gene by estrogen. J Steroid Biochem Mol Biol 2004; 91 : 225-239. 
11. Asselin-Labat ML, Biola-Vidamment A, Kerbrat S, Lombes M, Bertoglio J, Pallardy M. FoxO3 mediates antagonistic effects of glucocorticoids and interleukin-2 on glucocorticoidinduced leucine zipper expression. Mol Endocrinol 2005; 19: 1752-1764.

12. Chen W, Rgatsky I, Garabedan MJ. MED14 and MD1 dfferenall reglate target-specfic gene actvaton by the glcocorticod receptor. Mol Endocrnol 2006; 20: 560-572.

13. Van der Laan S, Sarabdjitsingh RA, Van Batenburg MF, Lachize SB, Li H, Dijkmans TF et al. Chromatin immunoprecipitation scanning identifies glucocorticoid receptor binding regions in the proximal promoter of a ubiquitously expressed glucocorticoid target gene in brain. J Neurochem 2008; 106: 2515-2523.

14. Ayroldi E, Migliorati G, Bruscoli S, Marchetti C, Zollo O, Cannarile L et al. Modulation of T-cell activation by the glucocorticoid-induced leucine zipper factor via inhibition of nuclear factor-êB. Blood 2001; 98: 743-753.

15. Delfino DV, Agostini M, Spinicelli S, Vito P, Riccardi C. Decrease of Bcl-xL expression and augmentation of apoptosis in GILZ-overexpressing transgenic mice. Blood 2004; 104: 4134-4141.

16. Ayroldi E, Zollo O, Bastianelli A, Marchetti C, Agostini M, Di Virgilio R et al. GILZ mediates the antiproliferative activity of glucocorticoids by negative regulation of Ras signaling. J Clin Invest 2007; 117: 1605-1615.

17. Cannarile L, Fallarino F, Agostini M, Cuzzocrea S, Mazzon E, Vacca C et al. Increased Gilz expression in transgenic mice up-regulates Th-2 lymphokines. Blood 2006; 107: 1039-1047.

18. Cannarile L, Cuzzocrea S, Santucci L, Agostini M, Mazzon E, Esposito E et al. Glucocorticoid-induced leucine zipper is protective in Th1-mediated models of colitis. Gastroenterology 2009; 136: 530-541.

19. Cohen N, Mouly E, Hamdi H, Maillot M, Pallardy M, Godot V et al. GILZ expression in human dendritic cells redirects their maturation and prevents antigen-specific $\mathrm{T}$ lymphocyte response. Blood 2006; 107: 2037-2044

20. Hamdi H, Godot V, Maillot MC, Prejean MV, Cohen N, Krzysiek R et al. Induction of antigen-specific regulatory $\mathrm{T}$ lymphocytes by human dendritic cells expressing the glucocorticoid-induced leucin zipper. Blood 2007; 110: 211-219.

21. Muller OG, Parnova RG, Centeno G, Rossier BC, Firsov D, Horisberger JD. Mineralcorticoid effects in the kidney: correlation between alphaENaC, GILZ, and Sgk-1 mRNA expression and urinary excretion of $\mathrm{Na}^{+}$and $\mathrm{K}^{+}$. J Am Soc Nephrol 2003; 14 1107-1115.

22. Soundararajan R, Zhang TT, Wang J, Vandewalle A, Pearce D. A novel role for glucocorticoid-induced leucine zipper protein in epithelial sodium channel-mediated sodium transport. J Biol Chem 2005; 280: 39970-39981.

23. Ayroldi E, Riccardi C. Glucocorticoid-induced leucine zipper (GILZ): a new important mediator of glucocorticoid action. FASEB J 2009; 23: 3649-3658.

24. Delfino DV, Agostini M, Spinicelli S, Vacca C, Riccardi C. Inhibited cell death, NF- $\kappa$ B activity and increased IL-10 in TCR-triggered thymocytes of transgenic mice overexpressing the glucocorticoid-induced protein GILZ. Int Immunopharmacol 2006; 6 : $1126-1134$.

25. Hochstrasser M. Origin and function of ubiquitin-like proteins. Nature 2009; 458: 422-429.

26. Bergink S, Jentsch S. Principles of ubiquitin and SUMO modifications in DNA repair. Nature 2009; 458: 461-467.

27. Mabb AM, Miyamoto S. SUMO and NF-kappaB ties. Cell Mol Life Sci 2007; 64: 1979-1996.

28. Kang TB, Ben-Moshe T, Varfolomeef EE, Pewzner-Jung Y, Yogev N, Jurewicz A et al. Caspase-8 serves both apoptotic and nonapoptotic roles. J Immunol 2004; 173 2976-2984.

29. Hennet T, Hagen FK, Tabak LA, Marth JD. T-cell-specific deletion of a polypeptide $N$ acetylgalactosaminyl-transferase gene by site-directed recombination. Proc Natl Acad Sci USA 1995; 92: 12070-12074

30. Lawrence CP, Chow SC. FADD deficiency sensitises Jurkat T cells to TNF- $\alpha$-dependent necrosis during activation-induced cell death. FEBS Lett 2005; 579: 6465-6472.

31. Herold MJ, McPherson KG, Reichardt HM. Glucocorticoids in T cell apoptosis and function. Cell Mol Life Sci 2006; 63: 60-72.

32. Reichardt HM, Kaestner KH, Tuckermann J, Kretz O, Wessely O, Bock R et al. DNA binding of the glucocorticoid receptor is not essential for survival. Cell 1998; 93: 531-541.

33. Tuckermann JP, Kleiman A, McPherson KG, Reichardt HM. Molecular mechanisms of glucocorticoids in the control of inflammation and lymphocyte apoptosis. Crit Rev Clin Lab Sci 2005; 42: 71-104

34. Distelhorst CW. Recent insights into the mechanism of glucocorticoid-induced apoptosis. Cell Death Differ 2002; 9: 6-19.

35. Frankfurt O, Rosen ST. Mechanisms of glucocorticoid-induced apoptosis in hematologic malignancies: updates. Curr Opin Oncol 2004; 16: 553-563.

36. Salmena L, Lemmers B, Hakem A, Matysiak-Zablocki E, Murakami K, Au PY et al. Essential role for caspase 8 in T-cell homeostasis and T-cell-mediated immunity. Genes Dev 2003; 17: 883-895.

37. Berger $A B$, Sexton KB, Bogyo M. Commonly used caspase inhibitors designed based on substrate specificity profiles lack selectivity. Cell Res 2006; 16: 961-963.

38. Zhu S, Sachdeva M, Wu F, Lu Z, Mo Y-Y. Ubc9 promotes breast cell invasion and metastasis in a sumoylation-independent manner. Oncogene 2009; 29: 1763-1772.

39. Sun X-M, Butterworth M, MacFarlane M, Dubiel W, Ciechanover A, Cohen GM. Caspase activation inhibits proteasome function during apoptosis. Mol Cell 2004; 14: 81-93.

40. Maelfait J, Beyaert R. Non-apoptotic functions of caspase-8. Biochem Pharmacol 2008; 76 1365-1373.

41. Tang J, Kawadler $\mathrm{H}$, Yang X. Lymphocyte life and death: the caspase-8 connection. Cancer Biol Ther 2005; 4: 700-702.

42. Su HC, Lenardo MJ. Genetic defects of apoptosis and primary immunodeficiency. Immunol Allergy Clin North Am 2008; 28: 329-351.

\section{Supplementary Information accompanies the paper on Cell Death and Differentiation website (http://www.nature.com/cdd)}

